\title{
Bounds for functions of dependent risks
}

\author{
Paul Embrechts $^{1}$, Giovanni Puccetti ${ }^{2}$ \\ ${ }^{1}$ Department of Mathematics ETHZ, CH-8092 Zurich, Switzerland, \\ e-mail:embrechts@math.ethz.ch \\ ${ }^{2}$ Department of Mathematics for Decisions University of Firenze, 50314 Firenze, Italy, \\ e-mail:giovanni.puccetti@dmd.unifi.it
}

\begin{abstract}
The problem of finding the best-possible lower bound on the distribution of a non-decreasing function of $n$ dependent risks is solved when $n=2$ or a lower bound on the copula of the portfolio is provided. In this paper we correct the statement and the proof of this result, given in Embrechts, Höing, and Juri (2003). The problem gets much more complicated in arbitrary dimensions when no information on the structure of dependence of the random vector is available. In this case we provide a bound on the distribution function of the sum of risks which we prove to be better than the one generally used in the literature.
\end{abstract}

Key words copulas - dependent risks - dependency bounds - Fréchet bounds

JEL Classification G10

Mathematics Subject Classification (2000) 60E15, 60E05

\section{Introduction}

Consider a $n$-variate real function $\psi$ and a random vector $X:=\left(X_{1}, \ldots, X_{n}\right)$. In this paper we study the problem of finding the best-possible lower bound on the distribution function (df) of $\psi(X)$ when the marginal distributions of the individual risks $X_{i}$ are given and the structure of dependence of $X$ is partially or completely unknown. This problem has a long history. Makarov (1981), in response to a question formulated by A.N. Kolmogorov, provided the first result for $n=2$ and $\psi=+$, the sum operator. Some years later Frank et al. (1987) restated Makarov's result, using the well-known formulation of the problem based on copulas. Independently from this $g e$ ometric approach, Rüschendorf (1982) gave a much more elegant proof of the same theorem using a dual result proved for a more general purpose. The dual approach of Rüschendorf was related to a much earlier issue, dating back to 1871: the so-called Monge mass-transportation problem; in particular, he solved a special case of its Kantorovich version. A complete analysis of this kind of problems is given in Rachev and Rüschendorf (1998). The use of dependence information to tighten the bound on the df of a two-dimensional portfolio firstly appeared in Williamson and Downs (1990), where sharpness was proved for non-decreasing functionals $\psi$. Denuit et al. (1999) extended the bound for the sum to arbitrary dimensions and provided some applications; see also Embrechts et al. (2002) for the relevance of these techniques within quantitative risk management. Finally, Embrechts et al. (2003) gave the most general 
theorem till now, stating sharpness of the bound in the presence of information for a larger class of functions $\psi$. The latter article however contains a gap in the main proof; in our paper, we revisit the proof and correct the statement of their main result. While the problem can be considered fully solved if a lower bound on the copula of the vector $X$ is available, the search is still open in the no-information scenario for $n>2$, even for the case of the sum. In this case, a bound for the $\mathrm{df}$ of the sum of risks can be obtained by the previously cited theorems, but it fails to be sharp whenever $n>2$. Exploiting the dual result of Rüschendorf we give a better bound which, though not proved to be sharp, improves considerably the previous estimate for the df of the sum of identically distributed risks. Numerical computations of the bound are also provided.

\subsection{Notation}

We first fix some notation. Given a vector $x=\left(x_{1}, \ldots, x_{n}\right) \in \mathbb{R}^{n}$ we write $x_{-i}:=$ $\left(x_{1}, \ldots, x_{i-1}, x_{i+1}, \ldots, x_{n}\right)$ to indicate the $(n-1)$-valued vector obtained from $x$ by deleting the $i$-th component. The indicator function of the set $B \subset \mathbb{R}$ is the function $1_{B}: \mathbb{R} \rightarrow \mathbb{R}$

$$
1_{B}(b):= \begin{cases}1 & \text { if } b \in B, \\ 0 & \text { otherwise. }\end{cases}
$$

Finally, for $k$ (possibly identical) real numbers $s_{1}, \ldots, s_{k}, U_{\left\{s_{1}, \ldots, s_{k}\right\}}: \mathbb{R} \rightarrow[0,1]$ denotes the df of a random variable uniformly distributed on $\left\{s_{1}, \ldots, s_{k}\right\}$.

\section{Definitions and preliminaries}

In this section we introduce the main mathematical problem and recall some wellknown concepts about copulas.

\subsection{Copulas as dependence structures}

Let $X_{1}, \ldots, X_{n}$ be $n$ real-valued random variables on some probability space $(\Omega, \mathfrak{A}, \mathbb{P})$, with given dfs $F_{i}(x)=\mathbb{P}\left[X_{i} \leq x\right], i=1, \ldots, n$. The random vector $X:=$ $\left(X_{1}, \ldots, X_{n}\right)$ can be seen as a portfolio of one-period financial or insurance risks. For some function $\psi: \mathbb{R}^{n} \rightarrow \mathbb{R}$, we consider the problem of bounding from below the distribution function of the random variable $\psi(X)$, over the class of possible dfs for $X$ having fixed marginals. In fact, we search for

$$
m_{\psi}(s):=\inf \left\{\mathbb{P}[\psi(X)<s]: X_{i} \backsim F_{i}, i=1, \ldots, n\right\} .
$$

Of course, the df of $\psi(X)$ can be computed once the function $F\left(x_{1}, \ldots, x_{n}\right)=\mathbb{P}\left[X_{1} \leq\right.$ $\left.x_{1}, \ldots, X_{n} \leq x_{n}\right]$ is known. The latter is uniquely defined through the marginal dfs and their interdependence. The tool for modelling these dependencies is offered by the concept of copula.

A copula is a $n$-dimensional df restricted to $[0,1]^{n}$ having standard uniform marginals. For a formal definition of copula, we refer to Nelsen (1999). Given a copula 
$C$ and a set of $n$ univariate marginals $F_{1}, \ldots, F_{n}$, one can always define a df $F$ on $\mathbb{R}^{n}$ having these marginals by

$$
F\left(x_{1}, \ldots, x_{n}\right):=C\left(F_{1}\left(x_{1}\right), \ldots, F_{n}\left(x_{n}\right)\right) .
$$

Sklar's theorem (see Sklar (1973, Th. 1)) states conversely that we can always find a copula $C$ coupling the marginals of a fixed df $F$ trough (2.2). For continuous marginal dfs, this copula is unique. In our set-up it is convenient to identify the df $F$ of $X$ with the copula $C$ merging the given marginals into the df $C\left(F_{1}\left(x_{1}\right), \ldots, F_{n}\left(x_{n}\right)\right)$. Denote by $\mu_{C}$ the corresponding probability measure on $\mathbb{R}^{n}$ and define:

$$
\begin{aligned}
\sigma_{C, \psi}\left(F_{1}, \ldots, F_{n}\right)(s): & =\mu_{C}[\psi(X)<s] \\
& =\int_{\{\psi<s\}} d C\left(F_{1}\left(x_{1}\right), \ldots, F_{n}\left(x_{n}\right)\right), \\
\tau_{C, \psi}\left(F_{1}, \ldots, F_{n}\right)(s): & =\sup _{x_{1}, \ldots, x_{n-1} \in \mathbb{R}} C\left(F_{1}\left(x_{1}\right), \ldots,\right. \\
& \left.F_{n-1}\left(x_{n-1}\right), F_{n}^{-}\left(\psi_{x_{-n}}^{-}(s)\right)\right),
\end{aligned}
$$

where $\widehat{\psi}_{x_{-n}}(s):=\sup \left\{x_{n} \in \mathbb{R}: \psi\left(x_{-n}, x_{n}\right)<s\right\}$ for fixed $x_{-n} \in \mathbb{R}^{n-1}$.

By the above discussion, problem (2.1) can be equivalently expressed as

$$
m_{\psi}(s)=\inf \left\{\sigma_{C, \psi}\left(F_{1}, \ldots, F_{n}\right)(s): C \in \mathfrak{C}_{n}\right\}
$$

where $\mathfrak{C}_{n}$ denotes the set of all $n$-dimensional copulas.

\subsection{Dependency information}

If we don't have the perfect knowledge of the copula $C$ coupling the fixed marginal dfs of the portfolio $X$, the df of $\psi(X)$ cannot be determined exactly and problem (2.5) arises. However, it can be the case that partial information regarding $C$ is known.

Given two copulas $C_{1}$ and $C_{2}$, we say that $C_{1} \geq$ (resp. $\leq$ ) $C_{2}$ if and only if $C_{1}(u) \geq$ (resp. $\left.\leq\right) C_{2}(u)$ for all $u \in[0,1]^{n}$. Using the properties of a copula it can be easily shown that any copula $C$ lies between the so-called lower and upper Fréchet bounds $W, M:[0,1]^{n} \rightarrow[0,1] ; W\left(u_{1}, \ldots, u_{n}\right):=\left(\sum_{i=1}^{n} u_{i}-n+1\right)^{+}$, $M\left(u_{1}, \ldots, u_{n}\right):=\min \left\{u_{1}, \ldots, u_{n}\right\}$, namely $W \leq C \leq M$. A third copula of interest is the product copula $\Pi:[0,1]^{n} \rightarrow[0,1], \Pi(u):=\prod_{i=1}^{n} u_{i}$ which represents independence among coupled random variables.

The copula of a df $F$ contains all the dependency information of $F$. Hence putting a lower bound on the copula $C$ of the portfolio can be interpreted as having partial information regarding its dependence structure. For instance, assuming that $C=M$ characterizes the risks of our portfolio as comonotonic, i.e. as being increasing functions of a common random variable. See Dhaene et al. (2002) for more details on comonotonicity. Moreover, assuming that $C \geq \Pi$ identifies the risks as positive lower orthant dependent (PLOD); see Nelsen (1999, Def. 5.6.1).

If we assume that a lower bound on $C$ is known, we can reduce our search to

$$
\begin{aligned}
m_{C_{L}, \psi}(s): & =\inf \left\{\sigma_{C, \psi}\left(F_{1}, \ldots, F_{n}\right)(s): C \geq C_{L}\right\} \\
& =\inf \left\{\mathbb{P}[\psi(X)<s]: X_{i} \backsim F_{i}, i=1, \ldots, n, F \geq C_{L}\left(F_{1}, \ldots, F_{n}\right)\right\} .
\end{aligned}
$$


Note that $m_{W, \psi}(t)=m_{\psi}(t)$, since assuming that $C \geq W$ corresponds to the situation in which we are completely ignorant about the dependence structure of the random vector $X$. Obviously, $m_{C_{L}, \psi}(t) \geq m_{\psi}(t)$ but we warn the reader that the last inequality is often strict even for a non-decreasing function $\psi$. Due to the fact that $\geq$ is not a complete ordering on $\mathfrak{C}_{n}$, letting $C \geq C_{L}$ is not necessarily a prudent assumption. In fact, for any $C_{L} \neq W$, we neglet all copulas which are not comparable to $C_{L}$ with respect to $\geq$. By doing so we possibly exclude the riskiest copula, i.e. the one possibly solving (2.5).

Finally note that, contrary to $M, W$ is not a copula for $n>2$; this fact will play a fundamental role in the next sections.

\section{Main result with partial information}

When partial information on the copula of a vector $X$ is known, it is easy to find a general lower bound on $\sigma_{C, \psi}\left(F_{1}, \ldots, F_{n}\right)(s)$.

Theorem 3.1. Let $X=\left(X_{1}, \ldots, X_{n}\right)$ be a random vector on $\mathbb{R}^{n}(n>1)$ having marginal distribution functions $F_{1}, \ldots, F_{n}$ and copula $C$. Assume that there exists a copula $C_{L}$ such that $C \geq C_{L}$. If $\psi: \mathbb{R}^{n} \rightarrow \mathbb{R}$ is non-decreasing in each coordinate, then for every real $s$ we have

$$
\sigma_{C, \psi}\left(F_{1}, \ldots, F_{n}\right)(s) \geq \tau_{C_{L}, \psi}\left(F_{1}, \ldots, F_{n}\right)(s) .
$$

Proof. First observe that for arbitrary $x \in \mathbb{R}^{n}$, the uniform continuity of a copula $C$ implies that

$$
\begin{aligned}
& \mu_{C}\left[X_{1} \leq x_{1}, \ldots, X_{n-1} \leq x_{n-1}, X_{n}<x_{n}\right] \\
& =\lim _{k \rightarrow \infty} \mu_{C}\left[X_{1} \leq x_{1}, \ldots, X_{n-1} \leq x_{n-1}, X_{n} \leq x_{n}-1 / k\right] \\
& =C\left(F_{1}\left(x_{1}\right), \ldots, F_{n-1}\left(x_{n-1}\right), \lim _{k \rightarrow \infty} F_{n}\left(x_{n}-1 / k\right)\right) \\
& =C\left(F_{1}\left(x_{1}\right), \ldots, F_{n-1}\left(x_{n-1}\right), F_{n}^{-}\left(x_{n}\right)\right) .
\end{aligned}
$$

Now fix $\left(\bar{x}_{1}, \ldots, \bar{x}_{n-1}\right) \in \mathbb{R}^{n-1}$ and assume that $\bar{x}_{n}:=\psi_{\widehat{\bar{x}}_{-n}}(s)$ is finite. Then

$$
\left\{X_{1} \leq \bar{x}_{1}, \ldots, X_{n-1} \leq \bar{x}_{n-1}, X_{n}<\bar{x}_{n}\right\} \subset\{\psi(X)<s\}
$$

and hence

$$
\begin{aligned}
\mu_{C}[\psi(X)<s] & \geq \mu_{C}\left[X_{1} \leq \bar{x}_{1}, \ldots, X_{n-1} \leq \bar{x}_{n-1}, X_{n}<\bar{x}_{n}\right] \\
& =C\left(F_{1}\left(\bar{x}_{1}\right), \ldots, F_{n-1}\left(\bar{x}_{n-1}\right), F_{n}^{-}\left(\bar{x}_{n}\right)\right) \\
& \geq C_{L}\left(F_{1}\left(\bar{x}_{1}\right), \ldots, F_{n-1}\left(\bar{x}_{n-1}\right), F_{n}^{-}\left(\widehat{\psi_{\bar{x}}}(s)\right)\right) .
\end{aligned}
$$

If $\bar{x}_{n}=+\infty$, then $\psi\left(\bar{x}_{-n}, x_{n}\right)<s$ for all $x_{n} \in \mathbb{R}$, and hence

$$
\begin{aligned}
\mu_{C}[\psi(X)<s] & \geq \mu_{C}\left[X_{1} \leq \bar{x}_{1}, \ldots, X_{n-1} \leq \bar{x}_{n-1}, X_{n} \in \mathbb{R}\right] \\
& =C\left(F_{1}\left(\bar{x}_{1}\right), \ldots, F_{n-1}\left(\bar{x}_{n-1}\right), 1\right) \\
& \geq C_{L}\left(F_{1}\left(\bar{x}_{1}\right), \ldots, F_{n-1}\left(\bar{x}_{n-1}\right), F_{n}^{-}(+\infty)\right) .
\end{aligned}
$$


Analogously, if $\bar{x}_{n}=-\infty$ then $\psi\left(\bar{x}_{-n}, x_{n}\right) \geq s$ for all $x_{n} \in \mathbb{R}$, so that

$$
\begin{aligned}
\mu_{C}[\psi(X)<s] \geq 0 & =C_{L}\left(F_{1}\left(\bar{x}_{1}\right), \ldots, F_{n-1}\left(\bar{x}_{n-1}\right), 0\right) \\
& =C_{L}\left(F_{1}\left(\bar{x}_{1}\right), \ldots, F_{n-1}\left(\bar{x}_{n-1}\right), F_{n}^{-}(-\infty)\right) .
\end{aligned}
$$

The theorem follows by taking the supremum over all $\left(\bar{x}_{1}, \ldots, \bar{x}_{n-1}\right) \in \mathbb{R}^{n-1}$.

We now prove that, if a lower copula-bound on $C$ is assumed, then there will always be a copula attaining bound (3.1), i.e. that bound cannot be tightened.

Theorem 3.2. In the hypotheses of Theorem 3.1 assume $\psi$ is also right-continuous in its last argument. Define the function $C_{t}:[0,1]^{n} \rightarrow[0,1]$ as follows:

$$
C_{t}(u):= \begin{cases}\max \left\{t, C_{L}(u)\right\} & \text { if } u=\left(u_{1}, \ldots, u_{n}\right) \in[t, 1]^{n}, \\ \min \left\{u_{1}, \ldots, u_{n}\right\} & \text { otherwise, }\end{cases}
$$

where $t=\tau_{C_{L}, \psi}\left(F_{1}, \ldots, F_{n}\right)(s)$. Then $C_{t}$ is a copula and it attains bound (3.1), i.e.

$$
\sigma_{C_{t}, \psi}\left(F_{1}, \ldots, F_{n}\right)(s)=t .
$$

Proof. We prove the theorem in two steps.

Lemma 3.1. The function $C_{t}$ is a copula.

Proof. According to Def. 2.10.6 in Nelsen (1999), we only have to show that $C_{t}$ is $n$ increasing on its domain. For this, if $a=\left(a_{1}, \ldots, a_{n}\right), b=\left(b_{1}, \ldots, b_{n}\right)$ are arbitrary vectors in $[0,1]^{n}$, with $a_{i} \leq b_{i}, i \in N:=\{1, \ldots, n\}$, we have to show that

$$
\sum_{j_{1}=1}^{2} \cdots \sum_{j_{n}=1}^{2}(-1)^{j_{1}+\cdots+j_{n}} C_{t}\left(u_{1 j_{1}}, \ldots, u_{n j_{n}}\right) \geq 0
$$

where $u_{i 1}=a_{i}, u_{i 2}=b_{i}$ for all $i \in N$. If $F_{i}\left(a_{i}\right) \geq t$ for all $i \in N$, then $C_{t}(u)=$ $C_{L}(u)$ for every $u \in \prod_{i=1}^{n}\left[a_{i}, b_{i}\right]$ and (3.3) follows from $n$-increasingness of $C_{L}$. Note that we can always find a permutation $\sigma: N \rightarrow N$ and $m \in N$ such that

$$
\begin{aligned}
F_{\sigma(1)}\left(a_{\sigma(1)}\right) \leq \cdots \leq F_{\sigma(m)}\left(a_{\sigma(m)}\right)<t \leq F_{\sigma(m+1)}\left(a_{\sigma(m+1)}\right) & \leq \cdots \\
& \leq F_{\sigma(n)}\left(a_{\sigma(n)}\right) .
\end{aligned}
$$

We prove the Lemma for $\sigma=\mathrm{Id}$, the identity function. By suitably exchanging summation indices in (3.3), the proof for a general $\sigma$ is obtained. Hence, we assume that

$$
F_{1}\left(a_{1}\right) \leq \cdots \leq F_{m}\left(a_{m}\right)<t \leq F_{m+1}\left(a_{m+1}\right) \leq \cdots \leq F_{n}\left(a_{n}\right)
$$

Observe that

$$
C_{t}\left(u_{11}, u_{2 j_{2}}, \ldots, u_{n j_{n}}\right)=F_{1}\left(a_{1}\right)
$$

for $j_{i}=1,2, i=2, \ldots, n$. Hence

$$
\sum_{j_{2}=1}^{2} \cdots \sum_{j_{n}=1}^{2}(-1)^{1+j_{2}+\cdots+j_{n}} C_{t}\left(u_{11}, u_{2 j_{2}}, \ldots, u_{n j_{n}}\right)=0
$$


the last expression being the sum of an even number of terms, all equal in absolute value but with alternate signs. Analogously, we have that

$$
C_{t}\left(u_{12}, u_{21}, u_{3 j_{3}} \ldots, u_{n j_{n}}\right)=\min \left\{F_{1}\left(b_{1}\right), F_{2}\left(a_{2}\right)\right\}
$$

for $j_{i}=1,2, i=3, \ldots, n$, and again

$$
\sum_{j_{3}=1}^{2} \cdots \sum_{j_{n}=1}^{2}(-1)^{1+j_{3}+\cdots+j_{n}} C_{t}\left(u_{12}, u_{21}, u_{3 j_{3}}, \ldots, u_{n j_{n}}\right)=0 .
$$

For $k=1, \ldots, m$ we can show that

$$
C_{t}\left(u_{12}, \ldots, u_{(k-1) 2}, u_{k 1}, u_{(k+1) j_{k+1}}, \ldots, u_{n j_{n}}\right)=\min \left\{\min _{1 \leq i \leq k-1} F_{i}\left(b_{i}\right), F_{k}\left(a_{k}\right)\right\}
$$

for all $j_{i}=1,2, i=k+1, \ldots, n$, and again

$$
\sum_{j_{k+1}=1}^{2} \cdots \sum_{j_{n}=1}^{2}(-1)^{1+j_{k+1}+\cdots+j_{n}} C_{t}\left(u_{12}, \ldots, u_{(k-1) 2}, u_{k 1}, u_{k+1 j_{k+1}}, \ldots, u_{n j_{n}}\right)=0 .
$$

By (3.4), the left-hand side of (3.3) reduces to

$$
\sum_{j_{m+1}=1}^{2} \cdots \sum_{j_{n}=1}^{2}(-1)^{j_{m+1}+\cdots+j_{n}} C_{t}\left(u_{12}, \ldots, u_{m 2}, u_{(m+1) j_{m+1}}, \ldots, u_{n j_{n}}\right) .
$$

If there exists $i \in\{1, \ldots, m\}$ so that $F_{i}\left(b_{i}\right)<t$ then, as before, (3.5) is zero because $F_{i}\left(a_{i}\right) \geq t, i=m+1, \ldots, n$. If instead $F_{i}\left(b_{i}\right) \geq t$, for all $i=1, \ldots, m$, then $C_{t}=C_{L}$ on the summation arguments in (3.5) and hence

$$
\begin{aligned}
& \sum_{j_{m+1}=1}^{2} \cdots \sum_{j_{n}=1}^{2}(-1)^{j_{m+1}+\cdots+j_{n}} C_{L}\left(u_{12}, \ldots, u_{m 2}, u_{(m+1) j_{m+1}}, \ldots, u_{n j_{n}}\right) \\
= & \mu_{C_{L}}\left[U_{1} \leq b_{1}, \ldots, U_{m} \leq b_{m}, U_{(m+1)} \in\left[a_{m+1}, b_{m+1}\right], \ldots, U_{n} \in\left[a_{n}, b_{n}\right]\right] \geq 0
\end{aligned}
$$

where $\left(U_{1}, \ldots, U_{n}\right) \backsim C_{L}$ on $[0,1]^{n}$. Since $a$ and $b$ are arbitrary, the lemma follows.

We now turn to the proof of Theorem 3.2.

First note that, since $\min \left\{u_{1}, \ldots, u_{n}\right\}$ yields the upper Fréchet bound, $C_{t} \geq C_{L}$. Hence by Theorem 3.1 we have

$$
\sigma_{C_{t}, \psi}\left(F_{1}, \ldots, F_{n}\right)(s) \geq t
$$

and it remains to prove the converse inequality. Consider the set

$$
B_{s}:=\left\{x \in \mathbb{R}^{n}: \psi(x)<s\right\} .
$$


If $t=1$, (3.6) leads to $\sigma_{C, \psi}\left(F_{1}, \ldots, F_{n}\right)(s)=1$ for every copula $C \geq C_{L}$. Consider $t \in[0,1)$ and assume that $B_{s}$ is non-empty (otherwise $\sigma_{C_{t}, \psi}\left(F_{1}, \ldots, F_{n}\right)(s)=0=t$ ). For an arbitrary $\tilde{x}=\left(\tilde{x}_{1}, \ldots, \tilde{x}_{n}\right) \in B_{s}, \psi(\tilde{x})<s$ and

$$
\psi_{\tilde{\tilde{x}}_{-n}}(s)=\sup \left\{x_{n} \in \mathbb{R}: \psi\left(\tilde{x}_{-n}, x_{n}\right)<s\right\} \geq \tilde{x}_{n} .
$$

If $\psi_{\widetilde{x}_{-n}}(s)=\tilde{x}_{n}$, then $\psi\left(\tilde{x}_{-n}, \tilde{x}_{n}+\epsilon\right) \geq s$ for all $\epsilon>0$, and by right-continuity of $\psi$ in its last argument, we obtain

$$
\psi(\tilde{x})=\lim _{\epsilon \rightarrow 0} \psi\left(\tilde{x}_{-n}, \tilde{x}_{n}+\epsilon\right) \geq s ;
$$

this contradicts the fact that $\tilde{x} \in B_{s}$. Hence $\psi_{\tilde{x}_{-n}}(s)>\tilde{x}_{n}$ and

$$
F_{n}^{-}\left(\psi_{\tilde{x}_{-n}}(s)\right)=\mu_{C_{t}}\left[X_{n}<\psi_{\tilde{x}_{-n}}(s)\right] \geq \mu_{C_{t}}\left[X_{n} \leq \tilde{x}_{n}\right]=F_{n}\left(\tilde{x}_{n}\right),
$$

which leads to

$$
\begin{aligned}
C_{L}\left(F_{1}\left(\tilde{x}_{1}\right), \ldots, F_{n}\left(\tilde{x}_{n}\right)\right) & \leq C_{L}\left(F_{1}\left(\tilde{x}_{1}\right), \ldots, F_{n}^{-}\left(\widehat{\hat{x}_{-n}}(s)\right)\right. \\
& \leq \sup _{x_{1}, \ldots, x_{n-1} \in \mathbb{R}} C_{L}\left(F_{1}\left(x_{1}\right), \ldots, F_{n}^{-}\left(\psi_{x_{-n}}(s)\right)\right)=t .
\end{aligned}
$$

From the definition of $C_{t}$, (3.7) implies that, for any $x \in B_{s}$,

$$
C_{t}\left(F_{1}\left(x_{1}\right), \ldots, F_{n}\left(x_{n}\right)\right)=\min \left\{t, F_{1}\left(x_{1}\right), \ldots, F_{n}\left(x_{n}\right)\right\} .
$$

Note that for $t=0$, we have $C_{0}\left(F_{1}\left(x_{1}\right), \ldots, F_{n}\left(x_{n}\right)\right)=0$, for all $x \in B_{s}$ and hence

$$
\sigma_{C_{0}, \psi}\left(F_{1}, \ldots, F_{n}\right)(s)=0 .
$$

We can then restrict to $t \in(0,1)$. Define now $x^{o}=\left(x_{1}^{o}, \ldots, x_{n}^{o}\right)$ by

$$
\begin{aligned}
x_{i}^{o} & :=\sup \left\{x_{i}: F_{i}\left(x_{i}\right)<t\right\}, i=1, \ldots, n-1, \\
x_{n}^{o} & :=\psi_{x_{-n}^{o}}(s)=\sup \left\{x_{n} \in \mathbb{R}: \psi\left(x_{-n}^{o}, x_{n}\right)<s\right\} .
\end{aligned}
$$

Note that $x_{i}^{o}$ is finite for $i=1, \ldots, n-1$ because the $F_{i}$ 's are (non-defective) distribution functions on $\mathbb{R}$ and

$$
x_{i}<x_{i}^{o} \Rightarrow F_{i}\left(x_{i}\right)<t \text {, for all } i=1, \ldots, n-1 .
$$

Moreover, right-continuity of the marginals implies that

$$
x_{i} \geq x_{i}^{o} \Rightarrow F_{i}\left(x_{i}\right) \geq t, \text { for all } i=1, \ldots, n-1 .
$$

This claim also holds for $i=n$. Indeed, suppose there exists $x_{n}^{\prime} \geq x_{n}^{o}=\widehat{\psi_{x_{-n}^{o}}}(s)$ such that $F_{n}\left(x_{n}^{\prime}\right)<t$, and fix an arbitrary vector $\left(x_{1}, \ldots, x_{n-1}\right) \in \mathbb{R}^{n-1}$. If $x_{i}<x_{i}^{o}$ for some $i=1, \ldots, n-1$ then $F_{i}\left(x_{i}\right)<t$ and

$$
\begin{aligned}
C_{L}\left(F_{1}\left(x_{1}\right), \ldots, F_{i}\left(x_{i}\right), \ldots, F_{n}^{-}\left(\widehat{\psi_{x_{-n}}}\right)\right) & \leq C_{L}\left(1, \ldots, 1, F_{i}\left(x_{i}\right), 1, \ldots, 1\right) \\
& \leq F_{i}\left(x_{i}\right)<t
\end{aligned}
$$


If instead $x_{i} \geq x_{i}^{o}$ for all $i=1, \ldots, n-1$, then $\widehat{\psi_{x_{-n}}} \leq \psi_{x_{-n}^{o}} \leq x_{n}^{\prime}$ and

$$
\begin{aligned}
C_{L}\left(F_{1}\left(x_{1}\right), \ldots, F_{n}^{-}\left(\widehat{\psi_{x_{-n}}}\right)\right) & \leq C_{L}\left(1, \ldots, 1, F_{n}^{-}\left(x_{n}^{\prime}\right)\right) \\
& \leq F_{n}^{-}\left(x_{n}^{\prime}\right)<t .
\end{aligned}
$$

Hence we have

$$
t=\sup _{x_{1}, \ldots, x_{n-1} \in \mathbb{R}} C_{L}\left(F_{1}\left(x_{1}\right), \ldots, F_{n}^{-}\left(\widehat{\psi_{x_{-n}}}\right)\right)<t,
$$

which is a contradiction and thus we can extend (3.10) to

$$
x_{i} \geq x_{i}^{o} \Rightarrow F_{i}\left(x_{i}\right) \geq t, \text { for all } i=1, \ldots, n .
$$

Combining (3.8), (3.9) and (3.12) we obtain, for all $x \in B_{s}$,

$$
\begin{aligned}
C_{t}( & \left.F_{1}\left(x_{1}\right), \ldots, F_{n}\left(x_{n}\right)\right) \\
= & \left\{\begin{array}{lr}
\min \left\{t, F_{1}\left(x_{1}\right), \ldots, F_{i-1}\left(x_{i-1}\right), F_{i+1}\left(x_{i+1}\right), \ldots, F_{n}\left(x_{n}\right)\right\} & \text { if } x_{i} \geq x_{i}^{o} \\
\min \left\{t, F_{1}\left(x_{1}\right), \ldots, F_{n-1}\left(x_{n-1}\right)\right\} & \text { for some } i \in\{1, \ldots, n-1\}, \\
\min \left\{F_{1}\left(x_{1}\right), \ldots, F_{n}\left(x_{n}\right)\right\} & \text { if } x_{n} \geq x_{n}^{o},
\end{array}\right.
\end{aligned}
$$

Now recall that

$$
\sigma_{C_{t}, \psi}\left(F_{1}, \ldots, F_{n}\right)=\mu_{C_{t}}\left[B_{s}\right]
$$

and consider the following covering of $B_{s}$

$$
B_{s} \subseteq \cup_{i=1}^{n} I_{i} \cup T
$$

where

$$
\begin{aligned}
I_{i} & :=\left\{x \in \mathbb{R}^{n}: x_{i}>x_{i}^{o}\right\} \cap B_{s}, i=1, \ldots, n, \\
T & :=\prod_{i=1}^{n}\left(-\infty, x_{i}^{o}\right] .
\end{aligned}
$$

Hence

$$
\mu_{C_{t}}\left[B_{s}\right] \leq \sum_{i=1}^{n} \mu_{C_{t}}\left[I_{i}\right]+\mu_{C_{t}}[T]=\mu_{C_{t}}[T]
$$

for $C_{t}$ is constant along the $i$-th dimension on $I_{i}$. If $x_{n}^{o}=+\infty$ then $\left(x_{-n}^{o}, x_{n}\right) \in B_{s}$ for all real $x_{n}$, and hence

$$
\begin{aligned}
\mu_{C_{t}}[T] & =\mu_{C_{t}}\left[X_{1} \leq x_{1}^{o}, \ldots, X_{n-1} \leq x_{n-1}^{o}\right] \\
& =\lim _{x_{n} \rightarrow+\infty} \mu_{C_{t}}\left[X_{1} \leq x_{1}^{o}, \ldots, X_{n-1} \leq x_{n-1}^{o}, X_{n} \leq x_{n}\right] \\
& =\lim _{x_{n} \rightarrow+\infty} C_{t}\left(F_{1}\left(x_{1}^{o}\right), \ldots, F_{n-1}\left(x_{n-1}^{o}\right), F_{n}\left(x_{n}\right)\right) \leq t .
\end{aligned}
$$


If instead $x_{n}^{o}$ is finite, observe that $\psi\left(x^{o}\right)=s$ by right-continuity of $\psi$ in the last argument, so it is sufficient to show that

$$
\mu_{C_{t}}\left[T \backslash\left\{x^{o}\right\}\right]=\mu_{C_{t}}\left[\cup_{i=1}^{n}\left\{X_{-i} \leq x_{-i}^{o}, X_{i}<x_{i}^{o}\right\}\right] \leq t .
$$

For $P \subset\{1, \ldots, n\}$ define

$$
A_{P}:=\left\{X_{i}<x_{i}^{o} \text { for } i \in P, X_{i} \leq x_{i}^{o} \text { for } i \in\{1, \ldots, n\} \backslash P\right\} .
$$

From elementary probability we have

$$
\begin{aligned}
& \mu_{C_{t}}\left[\cup_{i=1}^{n}\left\{X_{-i} \leq x_{-i}^{o}, X_{i}<x_{i}^{o}\right\}\right]=\mu_{C_{t}}\left[\cup_{i=1}^{n} A_{\{i\}}\right] \\
& =\sum_{1 \leq i_{1} \leq n}^{n} \mu_{C_{t}}\left[A_{\left\{i_{1}\right\}}\right]-\sum_{1 \leq i_{1} \leq i_{2} \leq n} \mu_{C_{t}}\left[A_{\left\{i_{1}, i_{2}\right\}}\right] \\
& +\sum_{1 \leq i_{1} \leq i_{2} \leq i_{3} \leq n} \mu_{C_{t}}\left[A_{\left\{i_{1}, i_{2}, i_{3}\right\}}\right]-\cdots+(-1)^{n+1} \mu_{C_{t}}\left[A_{\{1, \ldots, n\}}\right] .
\end{aligned}
$$

Observe that for every non-empty $P$

$$
A_{P}=\cup_{k \in \mathbb{N}} \cap_{i=1}^{n}\left\{X_{i} \leq x_{i}^{o}-(1 / k) 1_{P}(i)\right\},
$$

hence

$$
\mu_{C_{t}}\left[A_{P}\right]=\lim _{k \rightarrow \infty} C_{t}\left(F_{1}\left(x_{1}^{o}-(1 / k) 1_{P}(1)\right), \ldots, F_{n}\left(x_{n}^{o}-(1 / k) 1_{P}(n)\right)\right) .
$$

If $P \cap\{1, \ldots, n-1\} \neq \varnothing$ then $F_{i}\left(x_{i}^{o}-\frac{1}{k}\right)<t$ for some $i \in\{1, \ldots, n-1\}$ and all integers $k$; if instead $n \in P$ then $\left(x_{-n}^{o}, x_{n}^{o}-\frac{1}{k}\right) \in B_{s}$ for all integers $k$ by definition of $x_{n}^{o}$. These facts lead to

$$
\begin{aligned}
\mu_{C_{t}}\left[A_{P}\right] & =\lim _{k \rightarrow \infty} \min \left\{t, F_{1}\left(x_{1}^{o}-(1 / k) 1_{P}(1), \ldots, F_{n}\left(x_{n}^{o}-(1 / k) 1_{P}(n)\right)\right\}\right. \\
& =\min \left\{t, \min _{i \in P} F_{i}^{-}\left(x_{i}^{o}\right)\right\} .
\end{aligned}
$$

We assume, without loss of generality, that $F_{1}^{-}\left(x_{1}^{o}\right) \leq \cdots \leq F_{n-1}^{-}\left(x_{n-1}^{o}\right)$. Noting that (3.11) implies that $F_{n}^{-}\left(x_{n}^{o}\right) \geq t>F_{n-1}^{-}\left(x_{n-1}^{o}\right)$, we can calculate (3.14):

$$
\begin{aligned}
\mu_{C_{t}}\left[T \backslash\left\{u^{o}\right\}\right] & \\
= & \sum_{i=1}^{n-1} F_{i}^{-}\left(x_{i}^{o}\right)+\min \left\{t, F_{n}^{-}\left(x_{n}^{o}\right)\right\} \\
& -\left(\begin{array}{c}
n-1 \\
1
\end{array}\right) F_{1}^{-}\left(x_{1}^{o}\right)-\left(\begin{array}{c}
n-2 \\
1
\end{array}\right) F_{2}^{-}\left(x_{2}^{o}\right)-\cdots-\left(\begin{array}{l}
1 \\
1
\end{array}\right) F_{n-1}^{-}\left(x_{n-1}^{o}\right) \\
& +\left(\begin{array}{c}
n-1 \\
2
\end{array}\right) F_{1}^{-}\left(x_{1}^{o}\right)+\left(\begin{array}{c}
n-2 \\
2
\end{array}\right) F_{2}^{-}\left(x_{2}^{o}\right)+\cdots+\left(\begin{array}{l}
1 \\
1
\end{array}\right) F_{n-2}^{-}\left(x_{n-2}^{o}\right) \\
& -\cdots+(-1)^{n+1} F_{1}^{-}\left(x_{1}^{o}\right) .
\end{aligned}
$$

Rearranging all the terms, we obtain 


$$
\begin{aligned}
& \mu_{C_{t}}\left[T \backslash\left\{u^{o}\right\}\right] \\
& =F_{1}^{-}\left(x_{1}^{o}\right)\left[\left(\begin{array}{c}
n-1 \\
0
\end{array}\right)-\left(\begin{array}{c}
n-1 \\
1
\end{array}\right)+\left(\begin{array}{c}
n-1 \\
2
\end{array}\right)-\cdots+(-1)^{n-1}\left(\begin{array}{l}
n-1 \\
n-1
\end{array}\right)\right] \\
& +F_{2}^{-}\left(x_{2}^{o}\right)\left[\left(\begin{array}{c}
n-2 \\
0
\end{array}\right)-\left(\begin{array}{c}
n-2 \\
1
\end{array}\right)+\left(\begin{array}{c}
n-2 \\
2
\end{array}\right)-\cdots+(-1)^{n-2}\left(\begin{array}{l}
n-2 \\
n-2
\end{array}\right)\right] \\
& +\ldots \\
& +F_{n-1}^{-}\left(x_{n-1}^{o}\right)\left[\left(\begin{array}{l}
1 \\
0
\end{array}\right)-\left(\begin{array}{l}
1 \\
1
\end{array}\right)\right]+t .
\end{aligned}
$$

Recall that $\sum_{i=0}^{n}(-1)^{i}\left(\begin{array}{c}n \\ i\end{array}\right)=0$ for all integer $n$, hence (3.15) simplifies to

$$
\mu_{C_{t}}\left[T \backslash\left\{u^{o}\right\}\right]=t,
$$

which completes the proof.

Remark 3.1. There are several points worth noting regarding this theorem.

(i) For $n=2, C_{L}=W$ and $\psi(x)=x_{1}+x_{2}$ we get Proposition 1 in Rüschendorf (1982) and, in equivalent form, Theorem 1 in Makarov (1981) and Theorem 3.2 in Frank et al. (1987). In these papers, as well as in Embrechts et al. (2003), a sharp upper bound on the $\mathrm{df}$ of $\psi(X)$ is also given.

(ii) The theorem cannot be strengthened to read

$$
\mu_{C_{t}}[\psi(X) \leq t]=\sup _{x_{1}, \ldots, x_{n-1} \in \mathbb{R}} C\left(F_{1}\left(x_{1}\right), \ldots, F_{n-1}\left(x_{n-1}\right), F_{n}\left(\widehat{\psi}_{x_{-n}}(s)\right)\right),
$$

for $\sigma_{C, \psi}\left(F_{1}, \ldots, F_{n}\right)(s+):=\mu_{C}[\psi(X) \leq s]$ may have no minimum over the set $\mathfrak{C}_{n}$. Hence, contrary to Embrechts et al. (2003, p. 151), (2.3) is the correct way of defining the operator $\sigma$, if one wants to state Theorem (3.2) correctly. See Nelsen (1999, p. 187) for more details in the case of the sum of risks.

(iii) Note that $C_{t}$ is not the unique copula attaining the bound $t$ for we can always change it on $[0, t]^{n}$ by substituting for the upper Fréchet bound any other copula $C \geq C_{L}$.

(iv) The last part of the proof (from (3.13) on) is necessary only if all $F_{i}$ 's are discontinuous at $x^{o}$. Indeed, if there exists $F_{i}, i=1, \ldots, n-1$ which is continuous at $x_{i}^{o}$, then $F_{i}\left(x_{i}^{o}\right)=t$ and

$$
\mu_{C_{t}}[T]=\mu_{C_{t}}\left[X_{1} \leq x_{1}^{o}, \ldots, X_{n} \leq x_{n}^{o}\right] \leq \mu_{C_{t}}\left[X_{i} \leq x_{i}^{o}\right]=F_{i}\left(x_{i}^{o}\right)=t .
$$

If instead $F_{n}$ is continuous at $x_{n}^{o}$, then $\mu_{C_{t}}\left[X_{n}=x_{n}^{o}\right]=0$ and

$$
\begin{aligned}
\mu_{C_{t}}[T] & =\mu_{C_{t}}\left[X_{1} \leq x_{1}^{o}, \ldots, X_{n} \leq x_{n}^{o}\right] \\
& =\mu_{C_{t}}\left[X_{1} \leq x_{1}^{o}, \ldots, X_{n-1} \leq x_{n-1}^{o}, X_{n}<x_{n}^{o}\right] \\
& =\lim _{x_{n} \rightarrow x_{n}^{o}} \min \left\{t, F_{1}\left(x_{1}^{o}\right), \ldots, F_{n-1}\left(x_{n-1}^{o}\right), F_{n}^{-}\left(x_{n}\right)\right\} \leq t .
\end{aligned}
$$


To this extent our theorem generalizes Theorem 3 in Williamson and Downs (1990), where the case of multiple discontinuities was excluded. Note however that the theorem in Williamson and Downs (1990) holds for dfs on $\overline{\mathbb{R}}$ (defective dfs) also.

(v) The hypothesis of right-continuity in the last argument of the function $\psi$ is necessary to prove sharpness of the bound. Take for instance $n=2, X_{1} \backsim F_{1}=$ $U_{\left\{0, \frac{1}{2}, 1\right\}}, X_{2} \backsim F_{2}=U_{\{0,1,1\}}$ and $\psi\left(x_{1}, x_{2}\right)=1_{\left\{x_{1} \geq 1, x_{2}>1\right\}}$. Note that

$$
\psi\left(\cdot, x_{2}\right)= \begin{cases}0 & \text { if } x_{1}<1 \\ 1_{\left\{x_{2}>1\right\}} & \text { otherwise }\end{cases}
$$

is not right-continuous. We have

$$
\widehat{\psi_{x_{1}}}(1)= \begin{cases}+\infty & \text { if } x_{1}<1 \\ 1 & \text { otherwise }\end{cases}
$$

and then $\tau_{\psi, W}\left(F_{1}, F_{2}\right)(1)=\sup _{x_{1} \in \mathbb{R}}\left[F_{1}\left(x_{1}\right)+F_{2}^{-}\left(\widehat{\psi_{x_{1}}}(1)\right)-1\right]^{+}=\frac{2}{3}$. By Theorem 3.2 we should have that

$$
\mu_{\frac{2}{3}}\left[\psi\left(X_{1}, X_{2}\right)<1\right]=\frac{2}{3}
$$

but this is impossible because it is evident that $\psi\left(X_{1}, X_{2}\right)=0(<1) \mathbb{P}$-a.s. for every probability measure $\mathbb{P}$ having $F_{1}$ and $F_{2}$ as marginals. The reader can verify that the theorem works with $\hat{\psi}\left(x_{1}, x_{2}\right)=1_{\left\{x_{1}>1, x_{2} \geq 1\right\}}$. Finally, one can easily check that defining $\tau_{W, \psi}\left(F_{1}, F_{2}\right)(1):=\sup _{x_{1} \in \mathbb{R}}\left[F_{1}\left(x_{1}\right)+F_{2}\left(\widehat{\psi_{x_{1}}}(1)\right)-1\right]^{+}$in the above example does not lead to a more general result.

The proof of Theorem 3.2 provided in this paper is rather long and technical, but the elegant proof of the same theorem given in Embrechts et al. (2003) contains a gap. First of all note that, as we said in Remark 3.1 (ii), the correct statement of the theorem requires the definition of the operator $\sigma$ as given in (2.3). In Embrechts et al. (2003), and using our notation, the authors state that

In particular $\mu_{C_{t}}$ assigns mass $t$ to any set $\left[0, u_{1}\right] \times \cdots \times\left[0, u_{n}\right]$ such that $C_{L}\left(u_{1}, \ldots, u_{n}\right)=t$, whence $\mu_{C_{t}}\left[\left\{C_{L} \leq t\right\}\right]=t \ldots$

From this the theorem follows easily. This claim is not correct, since, even in the simplest case of two uniformly distributed risks and $C_{L}=W$, we have that $\mu_{C_{t}}[\{W \leq$ $t\}]=1$ for every $t \in[0,1]$. Hence the correct statement is

In particular $\mu_{C_{t}}$ assigns mass $t$ to any set $\left[0, u_{1}\right] \times \cdots \times\left[0, u_{n}\right]$ such that $C_{L}\left(u_{1}, \ldots, u_{n}\right)=t$, whence $\mu_{C_{t}}\left[\left\{C_{L} \leq t\right\}\right] \geq t \ldots$

This does not yield the theorem and a new proof is then required.

For applications of Theorem 3.2, including how to calculate numerically the bound for every choice of $F_{1}, \ldots, F_{n}$ and $C_{L}$, see Embrechts et al. (2003). 


\section{Main result without information on dependence}

The bound in (3.1) holds in arbitrary dimensions. However, when $n>2$ and we have no information regarding the dependence structure of the portfolio (vector) $X$, it may fail to be sharp. In fact, when $n>2$ and we take $W$ as lower bound instead of $C_{L}$, the function $C_{t}$ defined in Theorem 3.2 fails to be a copula.

\subsection{Mutually exclusive risks}

Actually, there is an important special case when the lower Fréchet bound is a proper df and hence sharpness of the bound still holds, also in the no-information scenario. In fact, Theorem 3.7 in Joe (1997), based on a previous result by Dall'Aglio (1972), gives a necessary and sufficient condition for $W\left(F_{1}, \ldots, F_{n}\right)$ to be a df having marginals $F_{1}, \ldots, F_{n}$.

Theorem 4.1. When $n>2, W\left(F_{1}, \ldots, F_{n}\right)$ is a df on $\mathbb{R}^{n}$ if and only if one of the following conditions holds:

(i) $\sum_{i=1}^{n} F_{i}\left(x_{i}\right) \leq 1$ for all $x \in \mathbb{R}^{n}$ s.t. $0<F_{i}\left(x_{i}\right)<1, i=1, \ldots, n$,

(ii) $\sum_{i=1}^{n} F_{i}\left(x_{i}\right) \geq n-1$ for all $x \in \mathbb{R}^{n}$ s.t. $0<F_{i}\left(x_{i}\right)<1, i=1, \ldots, n$.

An example of non-negative risks which satisfy one of the conditions of the above theorem and have df $W$ can be found in Dhaene and Denuit (1999). They form the class of so-called mutually exclusive risks, those risks that can be positive at most one at a time. In this special case, the bound stated in Theorem 3.1 is sharp for arbitrary finite $n$.

\subsection{Non-negative continuous and identically distributed risks}

Throughout the rest of the paper we will consider $C_{L}=W$. In this situation, the bound (3.1) is no longer sharp if $n>2$, and it is convenient to express (2.5) by a duality result given in Rüschendorf (1982):

$$
\begin{array}{r}
m_{\psi}(s)=1-\inf \left\{\sum_{i=1}^{n} \int f_{i} d F_{i}: f_{i} \text { are bounded measurable functions on } \mathbb{R}\right. \text { s.t. } \\
\left.\qquad \sum_{i=1}^{n} f_{i}\left(x_{i}\right) \geq 1_{[s,+\infty)}\left(\psi\left(x_{1}, \ldots, x_{n}\right)\right) \text { for all } x_{i} \in \mathbb{R}, i=1, \ldots, n\right\} .
\end{array}
$$

This dual optimization problem is very difficult to solve. The only explicit results known in the literature are given in Rüschendorf (1982) for the case of the sum of marginals being all uniformly or binomially distributed. Unfortunately, the dependence structure which solves (4.1) in the case of the sum of uniform marginals does not work in the general case, where the solution may depend upon the marginals chosen. This is in contrast to the case of the copula $C_{t}$, which satisfies (3.2) for all choices of $F_{1}, \ldots, F_{n}$. For that reason, below we restrict our attention to $\psi(x)=\sum_{i=1}^{n} x_{i}$ and 
set all marginal dfs equal to a common $\mathrm{df} F$, which we assume to be non-negative and continuous. In this situation (4.1) reads as

$$
\begin{gathered}
m_{\psi}(s)=1-\inf \left\{n \int f d F: f \text { bounded measurable function on } \mathbb{R}\right. \text { s.t. } \\
\left.\sum_{i=1}^{n} f\left(x_{i}\right) \geq 1_{[s,+\infty)}\left(\sum_{i=1}^{n} x_{i}\right) \text { for all } x_{i} \in[0,+\infty)^{n}, i=1, \ldots, n\right\} .
\end{gathered}
$$

It is easy to show that the bound stated in (3.1), which we call standard bound in the following, reduces to

$$
\tau_{W,+}(F, \ldots, F)(s)=[n F(s / n)-n+1]^{+}
$$

for every $s \geq n F^{-1}\left(\frac{F\left(x_{F}^{*}\right)+n-1}{n}\right), x_{F}^{*}:=\inf \left\{x \geq 0: F^{\prime}\right.$ is decreasing on $\left.[x,+\infty)\right\}$. For the numerical example given in Section 5 we obtain that (4.3) holds when $s \geq 4.39$ (resp. 12.26) for a $\log -\operatorname{Normal}(-0.2,1)($ resp. $\Gamma(3,1))$ df. Since every Pareto density is decreasing in its domain, (4.3) is valid for every non-negative $s$ in case of a Pareto portfolio; this example is for instance relevant for the measurement of operational risk, as can be seen from Moscadelli (2004).

We use (4.2) to provide a bound which is better (i.e. $\geq$ ) than the standard one ((4.3)).

Theorem 4.2. Let $F$ be a non-negative, continuous $d f$. If $F_{i}=F, i=1, \ldots, n$, then for every $s \geq 0$,

$$
m_{+}(s) \geq 1-n \inf _{r \in[0, s / n)} \frac{\int_{r}^{s-(n-1) r}(1-F(x)) d x}{s-n r} .
$$

Proof. For $r \in[0, s / n]$ define $\hat{f}_{r}: \mathbb{R} \rightarrow \mathbb{R}$ as follows:

$$
\hat{f}_{r}(x):= \begin{cases}0 & \text { if } x<r \\ \frac{x-r}{s-n r} & \text { if } r \leq x \leq s-(n-1) r \\ 1 & \text { otherwise }\end{cases}
$$

We prove that $\hat{f}_{r}$ is an admissible function in (4.2). Since $\hat{f}_{r}$ is non-negative, it is sufficient to show that we have $\sum_{i=1}^{n} \hat{f}_{r}\left(x_{i}\right) \geq 1$ when $\sum_{i=1}^{n} x_{i} \geq s$. If $x_{i} \geq s-(n-$ 1) $r$ for some $i=1, \ldots, n$, this trivially follows, so take $x_{1}, \ldots, x_{n} \in[0, s-(n-1) x]$ with $\sum_{i=1}^{n} x_{i} \geq s$. Define

$$
I:=\left\{i \leq n: x_{i} \geq r\right\}, \quad \bar{I}:=\{1, \ldots, n\} \backslash I
$$

and observe that we have

$$
\sum_{i \in I} x_{i} \geq s-\sum_{i \in \bar{I}} x_{i} \geq s-\#(\bar{I}) r .
$$


By definition of $\hat{f}_{r}$ it follows that

$$
\begin{aligned}
\sum_{i=1}^{n} \hat{f}_{r}\left(x_{i}\right)=\sum_{i \in I} \hat{f}_{r}\left(x_{i}\right)=\sum_{i \in I} \frac{x_{i}-r}{s-n r} & =\frac{\sum_{i \in I} x_{i}-(\# I) r}{s-n r} \\
& \geq \frac{s-((\# I)+(\# \bar{I})) r}{s-n r} \geq 1 .
\end{aligned}
$$

The theorem follows by checking that

$$
\int \hat{f}_{r}(x) d F(x)=1-\frac{\int_{r}^{s-(n-1) r} F(x) d x}{s-n r}
$$

and taking the infimum over all $r \in[0, s / n)$.

Remark 4.1. (i) Note that

$$
\lim _{r \rightarrow s / n}\left\{1-n \frac{\int_{r}^{s-(n-1) r}(1-F(x)) d x}{s-n r}\right\}=n F(s / n)-n+1,
$$

hence it follows that (4.4) is greater or equal than the standard lower bound given in (4.3) (and hence in (3.1)) for every threshold $s$ at which (4.3) is valid. In Section 5 we actually show that (4.4) is strictly greater than (4.3) in several cases of interest.

(ii) For $n=2$, (4.4) gives the sharp bound already stated in (3.1).

(iii) For $n>2$, the infimum in (4.4) can be easily calculated numerically by finding the zero-derivative point of its argument in the specified interval.

The assumptions under which Theorem 4.2 is valid, though considerable with respect to the setting of the previous sections, are consistent with most dfs $F$ and thresholds $s$ of actuarial/financial interest. In fact, such a difference in generality of results implicitly shows that the assumption in Theorems 3.1 and 3.2 of a non-trivial lower bound on the copula $C$ of the portfolio is very strong.

\section{Numerical results}

In this section we numerically compare the dual bound (4.4) with the standard bound (4.3).

\subsection{Computing numerically the best-possible bound}

When the exact value is not available, a good approximation for $m_{+}(s)$ can be found by solving two linear problems (LPs). We follow Williamson and Downs (1990) in defining the two dfs

$$
\underline{F}_{N}(x):=\frac{1}{N} \sum_{i=1}^{N} 1_{\left[q_{r},+\infty\right)}(x), \bar{F}_{N}(x):=\frac{1}{N} \sum_{i=0}^{N-1} 1_{\left[q_{r},+\infty\right)}(x) ;
$$


the jump points $q_{0}, \ldots, q_{N}$ are the quantiles of $F$ defined by $q_{0}:=\inf \operatorname{supp}(F), q_{N}:=$ $\sup \operatorname{supp}(F)$ and $q_{r}:=F^{-1}\left(\frac{r}{N}\right), r=1, \ldots, N-1$. In the applications to follow we will always take $q_{0}=0$ and $q_{N}=+\infty$. We have $\underline{F}_{N} \leq F \leq \bar{F}_{N}$, from which it follows that, for every real $s$

$$
\sigma_{C,+}\left(\underline{F}_{N}, \ldots, \underline{F}_{N}\right)(s) \leq \sigma_{C,+}(F, \ldots, F)(s) \leq \sigma_{C,+}\left(\bar{F}_{N}, \ldots, \bar{F}_{N}\right)(s) .
$$

Therefore

$$
\underline{m}_{+}(s) \leq m_{+}(s) \leq \bar{m}_{+}(s),
$$

where $\underline{m}_{+}(s)$ and $\bar{m}_{+}(s)$ are naturally defined as:

$$
\begin{aligned}
& \underline{m}_{+}(s):=\inf \left\{\mathbb{P}\left[\sum_{i=1}^{n} X_{i}<t\right]: X_{i} \backsim \underline{F}_{N}, i=1, \ldots, n\right\}, \\
& \bar{m}_{+}(s):=\inf \left\{\mathbb{P}\left[\sum_{i=1}^{n} X_{i}<t\right]: X_{i} \backsim \bar{F}_{N}, i=1, \ldots, n\right\} .
\end{aligned}
$$

Given that $\underline{F}_{N}$ is a (possibly defective) discrete $\mathrm{df}, \underline{m}_{+}(s)$ is the solution of the following LP:

$$
\begin{array}{r}
\underline{m}_{+}(s)=\min _{p_{j_{1}, \ldots, j_{n}}} \sum_{j_{1}=1}^{N} \cdots \sum_{j_{n}=1}^{N} p_{j_{1}, j_{2}, \ldots, j_{n}} 1_{(-\infty, t)}\left(\sum_{i=1}^{n} q_{j_{i}}\right) \text { subject to } \\
\left\{\begin{array}{ccc}
\sum_{j_{2}=1}^{N} \sum_{j_{3}=1}^{N} \cdots \sum_{j_{n}=1}^{N} p_{j_{1}, \ldots, j_{n}}=\frac{1}{N} & j_{1}=1, \ldots, N, \\
\sum_{j_{1}=1}^{N} \sum_{j_{3}=1}^{N} \cdots \sum_{j_{n}=1}^{N} p_{j_{1}, \ldots, j_{n}}=\frac{1}{N} & j_{2}=1, \ldots, N, \\
\cdots, & & \\
\sum_{j_{1}=1}^{N} \sum_{j_{2}=1}^{N} \cdots \sum_{j_{n}-1}^{N}=1 & p_{j_{1}, \ldots, j_{n}}=\frac{1}{N} \quad \begin{array}{l}
j_{n}=1, \ldots, N, \\
0 \leq p_{j_{1}, \ldots, j_{n}} \leq 1
\end{array} & \begin{array}{l}
j_{i}=1, \ldots, N, \\
i=1, \ldots, n .
\end{array}
\end{array}\right.
\end{array}
$$

The function $\bar{m}_{+}(s)$ is the solution of an analogous LP. Since for $N$ tending to infinity the dfs $\bar{F}_{N}$ and $\underline{F}_{N}$ converge to the original df $F$, calculating $m_{+}(s)$ with any given level of accuracy is a matter of solving (5.2) and the corresponding LP for $\bar{m}_{+}(s)$ with $N$ large enough. Unfortunately, that is not a trivial task. The dimension of the two LPs is $N^{n}$ rows (variables) per $n N$ columns (constraints) and, while the length of the interval $\left[\underline{m}_{+}(s), \bar{m}_{+}(s)\right]$ asymptotically decreases as $1 / N$, the computational time and the memory needed to solve the two LPs increase exponentially. Finally note that a numerical solution will truncate $\underline{F}_{N}$ at a certain finite value. The software used automatically sets this upper limit so that (5.1) is maintained.

\subsection{Plots of the best-possible bound}

In this section we illustrate the quality of the estimate of the sharp bound on $\sigma_{C, \psi}\left(F_{1}, \ldots, F_{n}\right)$ provided by the dual bound (4.4). Some dfs of actuarial and financial interest are considered for $F$. In Figure 5.1, standard ((4.3)) and dual ((4.4)) bounds for a portfolio of three Pareto-distributed risks are given. It is relevant to note that the dual 
bound is strictly greater than the standard one, in accordance with Remark 4.1 (i). Most importantly, the dual value always falls within the range $\left[\underline{m}_{+}(s), \bar{m}_{+}(s)\right]$, which we plot for some thresholds of interest. This range has been calculated by setting $N=180$. The two linear problems have been solved using ILOG CPLEX ${ }^{\circledR}$ C Callable Libraries. Note that switching to $n=4$ drastically lowers the quality of approximation to $N<60$. In Figure 5.1, the values of $\mu_{C}\left[X_{1}+X_{2}+X_{3}<s\right]$ in case of independent $(C=\Pi)$ and comonotonic $(C=M)$ scenarios are also given. For the calculation of the distribution of the sum of comononotic random variables note that, in case of a common marginal $F$, we have that $\mu_{M}\left[\sum_{i=1}^{n} X_{i}<s\right]=F(s / n)$, while the convolution is computed by iterated conditioning, i.e. $\mu_{\Pi}\left[\sum_{i=1}^{n} X_{i}<s\right]=\int d F\left(x_{n}\right) \ldots \int d F\left(x_{2}\right) F\left(s-\sum_{i=2}^{n} x_{i}\right)$.

In Figures 5.2 and 5.3 we do the same for Log-Normal and $\Gamma$ portfolios. We remark that the time of computation of (4.4) is not affected by the dimension of the portfolio.

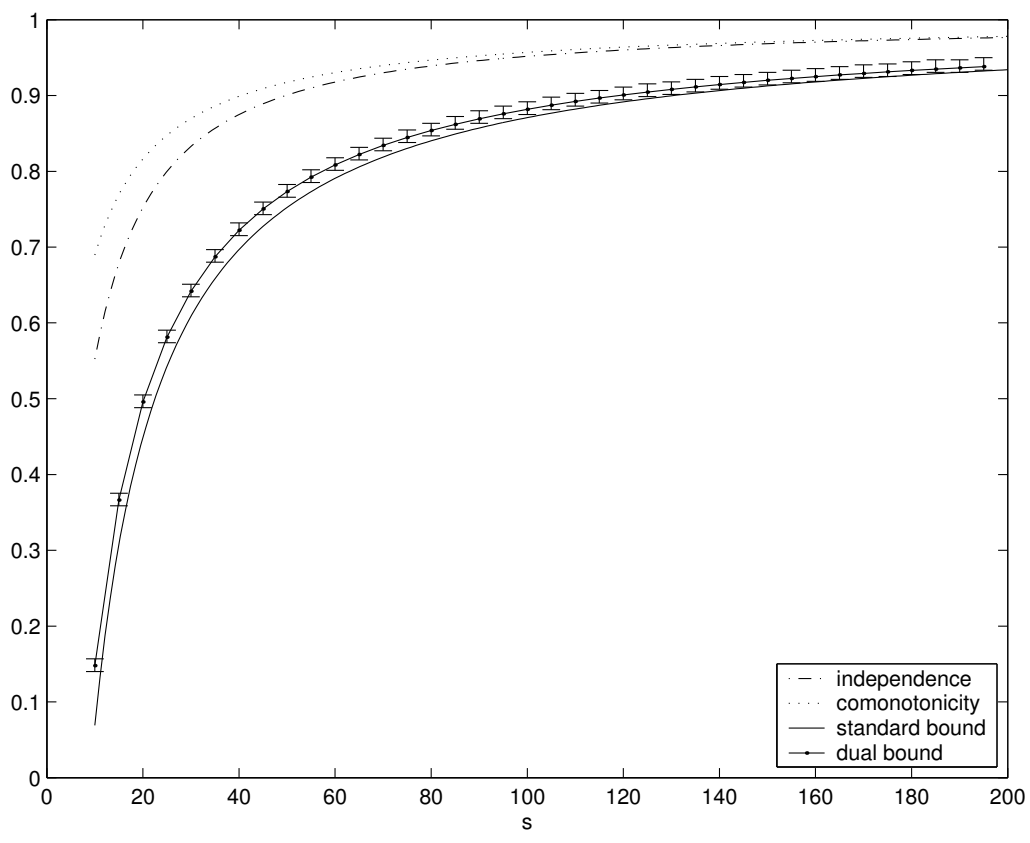

Figure 5.1: Range for $\mathbb{P}\left[X_{1}+X_{2}+X_{3}<s\right]$ for a Pareto(1.5,1)-portfolio under independence and comonotonic scenarios. We also give the standard ((4.3)) and dual ((4.4)) bounds. Numerical bounds for the true value of $m_{+}(s)$ are also given. 


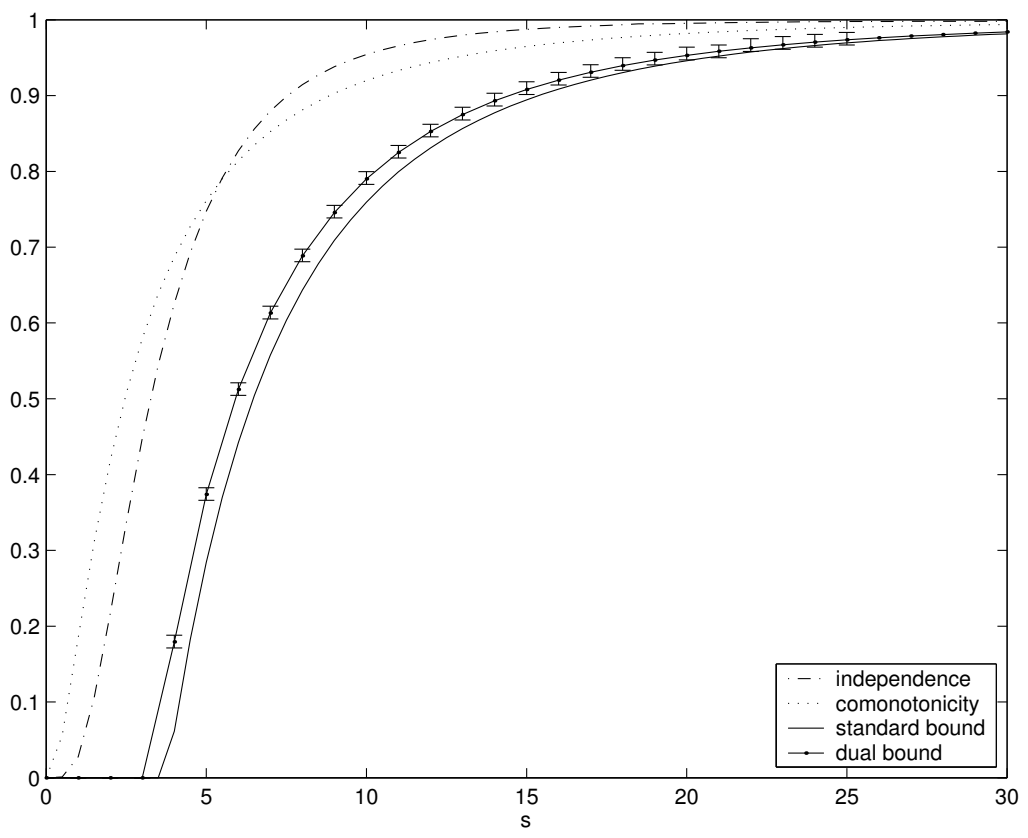

Figure 5.2: The same as Figure 1 for a Log-Normal(-0.2,1)-portfolio.

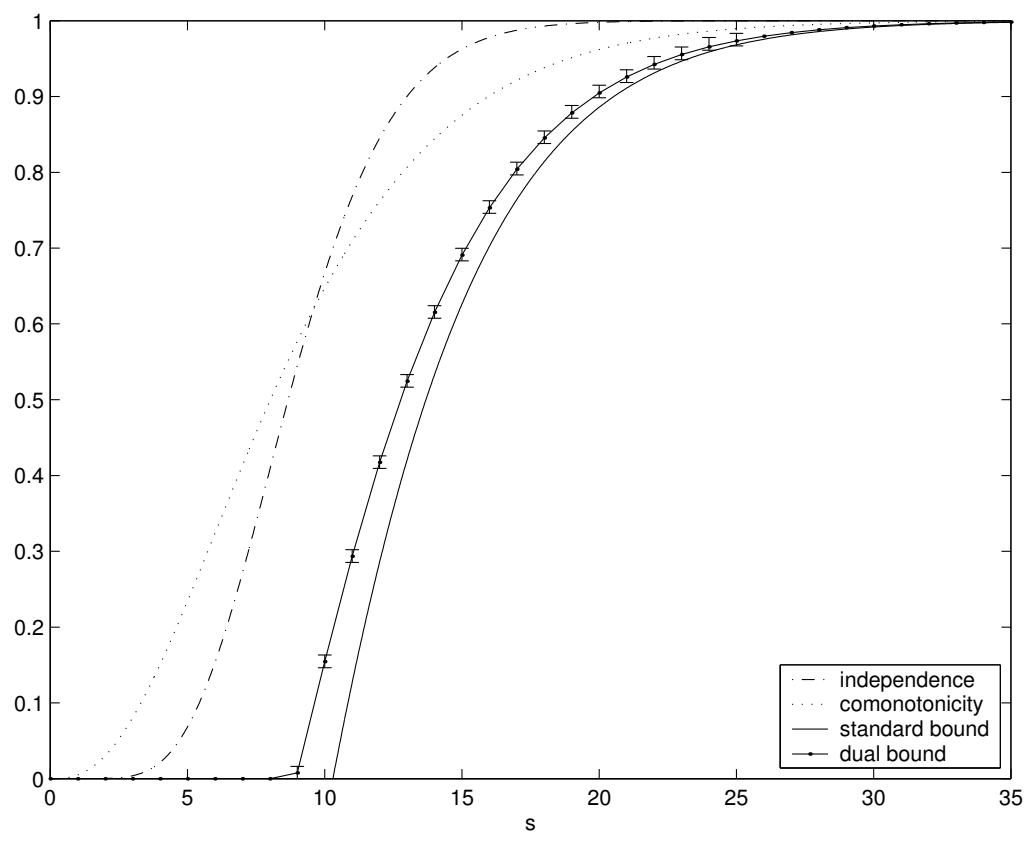

Figure 5.3: The same as Figure 1 for a $\Gamma(3,1)$-portfolio. 


\section{Conclusions}

The problem of finding the best-possible lower bound for the distribution of a nondecreasing function of dependent risks is solved when some information on the dependence structure of the portfolio is provided or the portfolio is two-dimensional. The problem gets much more complicated in arbitrary dimensions when no information on the copula of the random vector is given. In this case we provide a new bound which we prove to be better than the standard one generally used in the literature.

Acknowledgements. We would like to thank Ludger Rüschendorf and Andrea Höing for helpful discussions. The second author is grateful to the Department of Informatics, University of Pisa, for use of licensed software. He also gratefully acknowledges RiskLab at the ETH Zürich for financial support.

\section{References}

Cossette, H., M. Denuit, and É. Marceau (2002). Distributional bounds for functions of dependent risks. Schweiz. Aktuarver. Mitt. 1, 45-65.

Dall'Aglio, G. (1972). Fréchet classes and compatibility of distribution functions. Symposia Mathematica 9, 131-150.

Denuit, M., C. Genest, and É. Marceau (1999). Stochastic bounds on sums of dependent risks. Insurance Math. Econom. 25(1), 85-104.

Dhaene, J. and M. Denuit (1999). The safest dependence structure among risks. Insurance Math. Econom. 25(1), 11-21.

Dhaene, J., M. Denuit, M. J. Goovaerts, R. Kaas, and D. Vyncke (2002). The concept of comonotonicity in actuarial science and finance: theory. Insurance Math. Econom. 31(1), 3-33.

Embrechts, P., A. Höing, and A. Juri (2003). Using copulae to bound the value-atrisk for functions of dependent risks. Finance Stoch. 7(2), 145-167.

Embrechts, P., A. J. McNeil, and D. Straumann (2002). Correlation and dependence in risk management: properties and pitfalls. In M. Dempster (Ed.), Risk Management: Value at Risk and Beyond, pp. 176-223. Cambridge: Cambridge Univ. Press.

Frank, M. J., R. B. Nelsen, and B. Schweizer (1987). Best-possible bounds for the distribution of a sum-a problem of Kolmogorov. Probab. Theory Related Fields 74(2), 199-211.

Gaffke, N. and L. Rüschendorf (1981). On a class of extremal problems in statistics. Math. Operationsforsch. Statist. Ser. Optim. 12(1), 123-135.

Joe, H. (1997). Multivariate Models and Dependence Concepts. London: Chapman \& Hall.

Kellerer, H. G. (1984). Duality theorems for marginal problems. Z. Wahrsch. Verw. Gebiete 67(4), 399-432. 
Makarov, G. D. (1981). Estimates for the distribution function of the sum of two random variables with given marginal distributions. Theory Probab. Appl. 26, 803-806.

Moscadelli, M. (2004). The modelling of operational risk: experience with the analysis of the data collected by the Basel Committee. Preprint, Banca d'Italia.

Nelsen, R. B. (1999). An Introduction to Copulas, Volume 139 of Lecture Notes in Statistics. New York: Springer-Verlag.

Rachev, S. T. and L. Rüschendorf (1998). Mass Transportation Problems. Vol. I-II. Probability and its Applications (New York). New York: Springer-Verlag.

Rüschendorf, L. (1981). Sharpness of Fréchet-bounds. Z. Wahrsch. Verw. Gebiete 57(2), 293-302.

Rüschendorf, L. (1982). Random variables with maximum sums. Adv in Appl. Probab. 14(3), 623-632.

Sklar, A. (1973). Random variables, joint distribution functions, and copulas. Kybernetika (Prague) 9, 449-460.

Williamson, R. C. and T. Downs (1990). Probabilistic arithmetic. I. Numerical methods for calculating convolutions and dependency bounds. Internat. J. Approx. Reason. 4(2), 89-158. 